\title{
A Multiscale Decomposition Approach to Gel Image Interpretation
}

\author{
Xiaoran Mo and Roland Wilson \\ University of Warwick, Coventry CV4 7AL, UK \\ $\{$ xiaoran, rgw $\} @$ warwick.ac.uk
}

\begin{abstract}
Two-dimensional (2-D) polyacrylamide gel electrophoresis (PAGE) images are widely used in the area of Proteomics. In this paper, a region-based multiscale decomposition approach to gel image interpretation is presented. Instead of segmentation of each individual protein spot, the gel image is decomposed into small local regions with a quadtree structure. 2-D Gaussian functions are used to model local image features. Those local regional features will be used for further statistical analysis to extract discriminant features that distinguish different classes of gels. After a description of the method, experimental results are presented to show its potential in representing the protein related pixel intensities of the gel images.
\end{abstract}

\section{Introduction}

A two-dimensional (2-D) polyacrylamide gel electrophoresis (PAGE) image of a biological sample can be understood as a distribution profile of the proteins presented in the sample. The vertical and horizontal dimensions respectively reflect the molecular masses and isoelectric charges of the proteins, with the intensity of each pixel representing the amount of protein that migrated to that point.

Most of the current gel image interpretation methods are derived from the principle that a gel image consists of protein spots. Features are used to represent protein spots and a set of measurements are made from the image to specify each feature, which may involve its centroid, volume and other parameters. Existing methods for describing the gel image primitives can be roughly classified into two different approaches. The first approach, which avoids any assumption about the shape or internal structure of protein spots, searches for the location and/or boundary of each spot 123. Generally these methods detect protein spots by segmenting isolated spot regions with morphological algorithms. This means such methods are only implicit in their choice of spot model: it is hard to quantify their accuracy. Moreover, in practice, the problem of overlapping spots and over-segmentation occurs. The second approach, which relies on common characteristics, such as the shape of the spots, employs a parametric model to fit each spot 456,789. Though these methods give quantitative results on the modelling of spots, the complexity of the image data implies that the model may be inadequate. 
In our proposed method, there is no attempt to characterise each protein spot as a single feature. The gel image data is analysed through a region estimation process. Image regions are modelled as a sum of 2-D Gaussian functions, whose parameters are estimated locally. A quadtree-based image decomposition is employed to derive local image regions. Using the hierarchical decomposition approach, a quadtree structure will be generated for a gel image, with each node in the tree representing an image block at a certain scale. Gaussian functions are used to model the image data within each region. Without the detection of the location of each individual protein spot, the proposed method locates spot areas by means of measurements in local regions. Moreover, it allows for a controllable degree of fit to the image data, so that model complexity can be traded off against accuracy. After a brief account of the modelling process, results on real gel images are presented to show its effectiveness.

\section{The Model}

The local intensity profile in each gel image block is modelled by a separable 2-D Gaussian intensity function:

$$
g(x, y \mid \theta)=A \exp \left\{-\frac{1}{2}\left[\frac{\left(x-x_{0}\right)^{2}}{\sigma_{x}^{2}}+\frac{\left(y-y_{0}\right)^{2}}{\sigma_{y}^{2}}\right]\right\}
$$

parameterised by $\Theta=\left\{A, x_{0}, y_{0}, \sigma_{x}, \sigma_{y}\right\}$, where $A$ is the amplitude of the Gaussian; $\left(x_{0}, y_{0}\right)$ denotes its centre; and $\left(\sigma_{x}, \sigma_{y}\right)$ are the standard deviations in the horizontal and vertical axes, respectively.

Prior to the Gaussian modelling, the background is subtracted from the original pixel values. As the background detection is performed in local image regions, there will not be too much variation in the background intensities. A simple measure for the background intensity is used, which is the minimum pixel intensity within the image block, i.e.

$$
f_{b g}=\min _{\boldsymbol{x} \in B} f(\boldsymbol{x}) .
$$

where $B$ defines the set of pixels within the image block.

After the background subtraction, the image data are then windowed by a smooth function, to avoid edge effects. The set of parameters $\Theta=$ $\left\{A, x_{0}, y_{0}, \sigma_{x}, \sigma_{y}\right\}$ for data fitting using 2-D Gaussian functions is estimated by an iterative Maximum Likelihood (ML) approach [10].

$$
\begin{gathered}
A_{t+1}=\frac{\sum_{\boldsymbol{x}} f_{w}(\boldsymbol{x}) g\left(\boldsymbol{x} \mid \Theta_{t}\right)}{\sum_{\boldsymbol{x}} g\left(\boldsymbol{x} \mid \Theta_{t}\right) g\left(\boldsymbol{x} \mid \Theta_{t}\right)}, \quad \boldsymbol{\mu}_{t+1}=\frac{\sum_{\boldsymbol{x}} \boldsymbol{x} f_{w}(\boldsymbol{x}) g\left(\boldsymbol{x} \mid \Theta_{t}\right)}{\sum_{\boldsymbol{x}} f_{w}(\boldsymbol{x}) g\left(\boldsymbol{x} \mid \Theta_{t}\right)}, \\
\Sigma_{t+1}=\frac{2 \sum_{\boldsymbol{x}}\left(\boldsymbol{x}-\boldsymbol{\mu}_{t}\right)^{2} f_{w}(\boldsymbol{x}) g\left(\boldsymbol{x} \mid \Theta_{t}\right)}{\sum_{\boldsymbol{x}} f_{w}(\boldsymbol{x}) g\left(\boldsymbol{x} \mid \Theta_{t}\right)}
\end{gathered}
$$


where $\boldsymbol{x}=\left[\begin{array}{l}x \\ y\end{array}\right], \boldsymbol{\mu}=\left[\begin{array}{l}x_{0} \\ y_{0}\end{array}\right]$ and $\Sigma=\left[\begin{array}{cc}\sigma_{x}^{2} & 0 \\ 0 & \sigma_{y}^{2}\end{array}\right] ; f_{w}$ is the windowed image data; $g$ is the Gaussian function and $\Theta_{t}$ denotes the estimated parameter set from previous iteration.

For the initial estimate at iteration $t=0$, the centroid and variance of the Gaussian intensity profile are calculated as

$$
\boldsymbol{\mu}_{0}=\frac{\sum_{\boldsymbol{x}} \boldsymbol{x} f_{w}(\boldsymbol{x})}{\sum_{\boldsymbol{x}} f_{w}(\boldsymbol{x})}, \quad \Sigma_{0}=\frac{2 \sum_{\boldsymbol{x}}\left(\boldsymbol{x}-\boldsymbol{\mu}_{0}\right)^{2} f_{w}(\boldsymbol{x})}{\sum_{\boldsymbol{x}} f_{w}(\boldsymbol{x})} .
$$

The hierarchical quadtree decomposition procedure generates a set of quadrant nodes with a top-down approach, which successively subdivides the original frame by a factor of four. The subdivision continues until the block size reaches a preset minimum value. At each level, the image is divided into half-overlapping blocks, each windowed by the windowing function.

The windowing function is based on a squared cosine function, and is adaptive to the level of the subimage in the quadtree. If the depth of the quadtree is $D$, then the windowing function at the $k$ th level $(1 \leq k \leq D-1)$ is given as:

$$
w_{k}\left(x, y \mid x_{0}, y_{0}\right)= \begin{cases}w_{k}^{\prime}\left(x, y \mid x_{0}, y_{0}\right) & \text { if }\left|x-x_{0}\right| \leq \frac{N_{k}+N_{D}}{2} \text { and }\left|y-y_{0}\right| \leq \frac{N_{k}+N_{D}}{2} \\ 0 & \text { otherwise. }\end{cases}
$$

and

$$
w_{k}^{\prime}\left(x, y \mid x_{0}, y_{0}\right)=\sum_{i, j=0}^{2^{D-k}-1} w_{D}\left(x, y \mid x_{0}-\frac{N_{k}-N_{D}}{2}+i N_{D}, y_{0}-\frac{N_{k}-N_{D}}{2}+j N_{D}\right)
$$

with

$$
w_{D}\left(x, y \mid x_{0}, y_{0}\right)=\cos ^{2}\left(\frac{x-x_{0}}{2 N_{D}} \pi\right) \cos ^{2}\left(\frac{y-y_{0}}{2 N_{D}} \pi\right),
$$

where $\left(x_{0}, y_{0}\right)$ is the centre of the image block; $w_{D}$ is the windowing function for the bottom level, whose size is $2 N_{D}$ by $2 N_{D}$; the window size at the $k$ th level is $2 N_{k} \times 2 N_{k}$, and $N_{k}=2^{D-k} N_{D}$. While the windowing function at the bottom level, $w_{D}$, is a 2-D squared cosine function, for the levels above, a mixture of the squared cosine functions is used. For any two neighbouring quadrant regions in different levels of the quadtree, the window coefficients in the overlapping area will sum to one.

Before applying the Gaussian function to model the image data, a criterion called the information criterion is set to identify the "inactive" regions within the gel image, which are defined as background regions. The measure for this criterion is the total squared error when the image data is modelled by using the (constant) background intensity alone:

$$
M_{s r}=\sum_{\boldsymbol{x} \in B}\left(f(\boldsymbol{x})-f_{b g}\right)^{2} .
$$


If the value of $M_{s r}$ is above a given threshold, which is the minimum tolerance for a spot region, the criterion is met and the block is considered as a spot region; otherwise, a background block. Note that the background is calculated before applying the windowing function.

If an image block is classified as a spot region, the background intensity is subtracted from the image data, which is then multiplied by the windowing function. The windowed image data is fitted by the 2-D Gaussian function as described above. The Gaussian fitting is measured by the normalised residual sum of squares (NRSS), given as:

$$
N R S S_{i j}=\frac{\sum_{\boldsymbol{x} \in B_{i j}}\left\{f_{w}(\boldsymbol{x})-g\left(\boldsymbol{x} \mid \Theta_{i j}\right)\right\}^{2}}{\sum_{\boldsymbol{x} \in B_{i j}} f_{w}^{2}(\boldsymbol{x})}
$$

where $i j$ denotes the image block, $B_{i j}$ indicates the set of coordinates in the $i j$ th block and $\Theta_{i j}$ is the estimated set of Gaussian parameters for that image data.

The residual error of the synthesised data from the estimation is calculated for each image block at each block scale. A tree is built, with the nodes in each level indicating image blocks of the same scale and each node storing the residual error for the estimation. The multiscale approach is designed to retain the best estimation among all different image block scales. To determine which nodes are retained, a region node selection procedure is used. For every node at level $k$, $1 \leq k \leq D-1$ ( $D$ th level is the bottom level), the residual error obtained from this node (as parent node) is compared to the residual error of synthesised data constructed from the region measures in its four child nodes. The residual error for the node $(i, j)$ at level $k$ (as parent node) is given as

$$
e_{p}(i, j, k)=\sum_{\boldsymbol{x} \in B_{i j}}\left\{R(\boldsymbol{x}, i, j, k)-f_{w}(\boldsymbol{x})\right\}^{2}
$$

where $0<i, j<\frac{N}{N_{k}}+1$ with image size of $2 N \times 2 N ; R(\boldsymbol{x}, i, j, k)$ is the synthesised data value at coordinate $\boldsymbol{x}$ at $k$ th level for node $(i, j)$ :

$$
R(\boldsymbol{x}, i, j, k)= \begin{cases}0 & \text { for background node } \\ g(\boldsymbol{x}, i, j, k) & \text { for spot region node }\end{cases}
$$

The residual error of the synthesised data from the four children nodes at level $k+1$ is calculated as

$$
e_{c}(i, j, k)=\sum_{\boldsymbol{x} \in B_{i j}}\left\{\sum_{m, n=0}^{1} R(\boldsymbol{x}, 2 i-m, 2 j-n, k+1)-f_{w}(\boldsymbol{x})\right\}^{2}
$$

If $e_{p}(i, j, k)$ is less than $e_{c}(i, j, k)$, the Gaussian model parameters and background estimation at the parent level will be used to describe the region of image data; otherwise, the child nodes will be used. 


\section{Experiments}

The region modelling of gel images using the proposed method will be compared with the parametric spot modelling using the Gaussian spot model. The two gel images (Fig. 1) used in the experiments are from two different sample tissues. The images are 16-bit TIFF format images with zero-intensity pixels in white. Each image is of size $256 \times 256$ pixels.

The implementation of the spot modelling method using the Gaussian model is as follows: first a low-pass filter is applied to the original image to remove high frequency noise; secondly a peak detection algorithm is applied to the filtered image, and the detected local peaks are taken as the centres of the 2-D Gaussian models; finally the Gaussian model parameters are estimated. The local maxima are located by looking at the neighbouring pixels within a 5 by 5 window to see if the value of the central pixel is the largest and more than a given threshold above the average pixel value within the $5 \times 5$ neighbourhood. An iterative ML approach as described in section 2 is used to derive the parameter estimates. The detected spot centre coordinates give the initial estimate of the centres of the Gaussians. For both the initial estimates of $\sigma_{x}$ and $\sigma_{y}$, a value of 2 is used. The support region for the parameter estimation of each Gaussian model is defined as being an area of $\pm 3 \sigma_{x}$ and $\pm 3 \sigma_{y}$ from the spot centre in the horizontal and vertical directions. Fig. 2 shows the synthesised images from the Gaussian spot modelling method. As there is no information available about the actual proteins that are present in the gel image, the comparison is made from the calculated residual error of the resulting synthesised image.

The multiscale decomposition method produces an image representation with a tree structure, where there are two different types of leaf nodes in the quadtree: background nodes and spot nodes. To construct a synthesised gel image from the extracted measures, two different interpolation functions are defined. The function for the background node copies the background intensity value to each pixel in the corresponding block; while the function for the spot region node adds the Gaussian intensity value at the pixel position to the background value for each pixel.

A three-level quadtree structure is employed to derive local image regions, with a minimum block size of $8 \times 8$. A 2 -D Gaussian function, $S_{\text {model }}$, is defined to model the spot of minimum volume. The Gaussian spot model, $S_{\text {model }}$, has a standard deviation of 2 pixels respectively in the $x$ and $y$ directions, and the peak intensity in the spot region is 300 . The minimum value of $M_{s r}$ for an image block to be accepted as a spot region block is set at 1100000 , which is calculated from the Gaussian spot model, $\sum_{\boldsymbol{x}} S_{\text {model }}(\boldsymbol{x})^{2}$.

The result of the multiscale decomposition approach is shown in Fig. 3 As can be seen, the proposed region modelling method gives a smaller $N R S S$ values (around 0.07) than the parametric Gaussian spot modelling method, which gives an $N R S S$ value of around 0.15 for both images. 


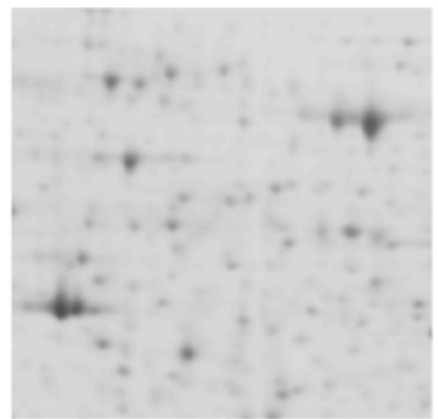

(a)

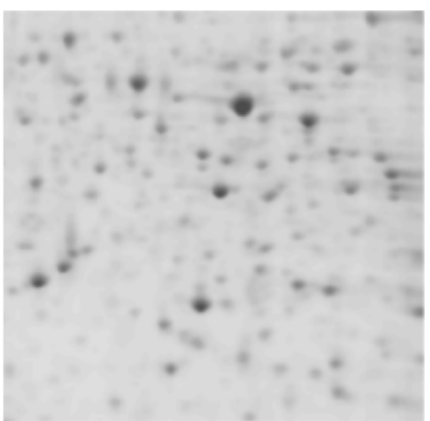

(b)

Fig. 1. Two examples of gel images: (a) Gel A and (b) Gel B.

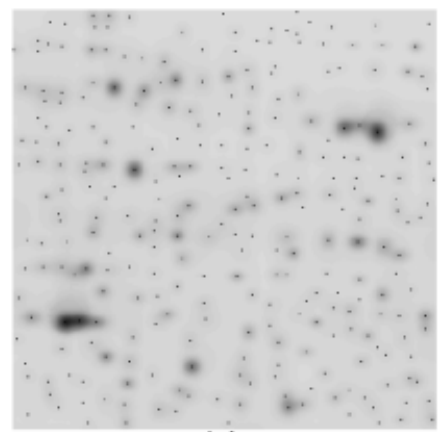

(a)

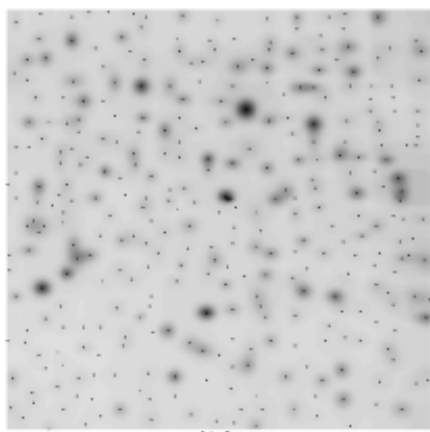

(b)

Fig. 2. The result of applying the parametric spot modelling method using Gaussian models: (a) the synthesised image of Gel A and (b) the synthesised image of Gel B (a black dot is used to indicate the Gaussian centre). NRSS value: 0.1495 (Gel A) and 0.1518 (Gel B); number of Gaussians: 280 (Gel A) and 334 (Gel B).

\section{Conclusions}

Existing gel image interpretation methods either employ low-level image processing techniques, such as background subtraction and peak detection by morphological filters, or are based on protein spot features, which describe each protein spot as one feature using some modelling function such as a 2-D Gaussian function. While it is impossible for the former methods to quantify their accuracy in extracting the significant information from the data, the latter methods rely on the detection of protein spot centres and boundaries, neither of which is easy. For parametric spot modelling methods which give quantitative results, it is very likely that the model is inadequate due to the complexity of the image data.

The advantage of the proposed region modelling method over the featurebased spot detection methods is that it avoids the segmentation of each indi- 


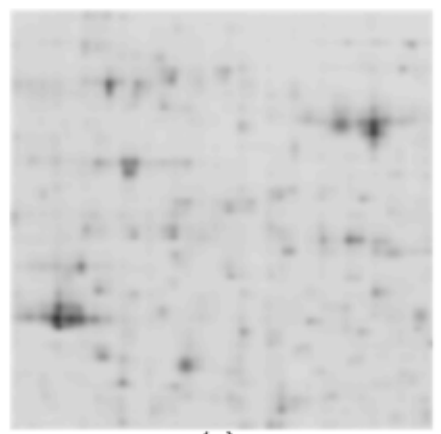

(a)

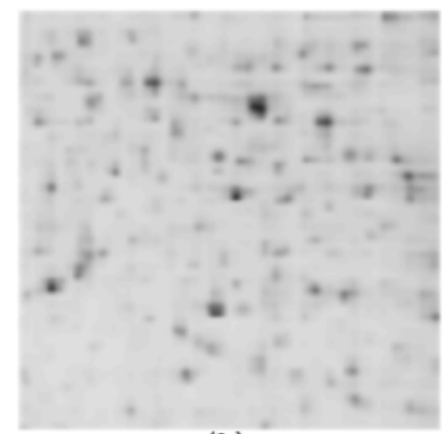

(b)

Fig. 3. The result of the multiscale decomposition approach: (a) the synthesised image of Gel A and (b) the synthesised image of Gel B. NRSS value: 0.074 (Gel A) and 0.072 (Gel B); number of splits: 1288 (Gel A) and 1308 (Gel B); number of Gaussians: 933 (Gel A) and 975 (Gel B).

vidual spots and any assumptions about the shape of the spots. As the image decomposition procedure does not result in every whole protein spot being captured in one image block, it is likely that some spots, especially large spots, lie within a number of connected blocks, and the spot is then approximated by a sum of Gaussians, each from one block. In such cases, further processing might be required to prepare the data for a conventional database. However, such problems can be avoided by applying discriminant analysis directly to the image data in the form of the regional features extracted using the proposed method, instead of using traditional techniques based on a database of protein spot features. This is an area which is being further researched and will be presented in due course.

From the normalised residual error values, it has been shown that the proposed method gives better results in representing the real image data in terms of the residual error of the synthesised data from the estimated image measures. Further work is in progress to compare the new representation with the conventional ones in the multivariate analysis of PAGE image datasets. We are also exploring other criteria for the region splitting process.

\section{References}

1. Lipkin, L.E., Lemkin, P.F.: Data-Base Techniques for Multiple Two-Dimensional Polyacrylamide Gel Electrophoresis Analyses. Journal of Clinical Chemistry, Vol. 26, No. 10 (1980) 1403-1412.

2. Skolnick, M.M.: Application of Morphological Transformations to the Analysis of Two-Dimensional Electrophoretic Gels of Biological Materials. Computer Vision, Graphics, and Image Processing, Vol. 35 (1986) 306-332. 
3. Pleißner, K.P., Hoffmann, F., Kriegel, K., Wenk, C., Wegner, S., Sahlström, A., Oswald, H., Alt, H., Fleck, E.: New Algorithm Approaches to Protein Spot Detection and Pattern Matching in Two-Dimensional Electrophoresis Gel Databases. Electrophoresis, Vol. 20 (1999) 755-765.

4. Anderson, N.L., Taylor, J., Scandora, A.E., Coulter, B.P., Anderson, N.G.: The TYCHO System for Computer Analysis of Two-Dimensional Gel Electrophoresis Patterns. Clinical Chemistry, Vol. 27, No. 11 (1981) 1807-1820.

5. Brown, W.T., Ezer, A.: A Computer Program Using Gaussian Fitting for Evaluation of Two-Dimensional Gels. Journal of Clinical Chemistry, Vol. 28, No. 4 (1982) 1041-1044.

6. Garrels, J.I.: The QUEST System for Two-Dimensional Gels. Journal of Biological Chemistry, Vol. 264, No. 9 (1989) 5269-5282.

7. Monardo, P.J., Boutell, T., Garrels, J.I., Latter, G.I.: A Distributed System for Two-Dimensional Gel Analysis. Journal of Computer Applications in the Biosciences (CABIOS), Vol. 10, No. 2 (1994) 137-143.

8. Bettens, E., Scheunders, P., Sijbers, J., Van Dyck, D., Moens, L.: Automatic Segmentation and Modelling of Two-Dimensional Electrophoresis Gels. Proceedings IEEE International Conference on Image Processing (1996) 665-668.

9. Wilson, R.: Modelling of 2D Gel Electrophoresis Images for Proteomics Databases. Proceedings on the 16th International Conference on Pattern Recognition (2002) 767-770.

10. Bhalerao, A., Wilson, R.: Estimating Local and Global Structure using a Gaussian Intensity Model. Proceedings of Medical Image Understanding and Analysis (MIUA) (2001). 